\title{
Analysis of Encoding and Decoding Techniques for the Interference Channel with Destination Cooperation
}

\author{
Ahmad Abu Al Haija \\ Department of Electrical \& Computer Engineering \\ McGill University, Montreal, QC, Canada \\ Email: ahmad.abualhaija@mail.mcgill.ca
}

\author{
Mai $\mathrm{Vu}$ \\ Department of Electrical \& Computer Engineering \\ Tufts University, Medford, MA, USA \\ Email:maivu@ece.tufts.edu
}

\begin{abstract}
In this paper, we analyze and compare several encoding and decoding techniques for the interference channel with destination cooperation (IC-DC). For the encoding techniques, we consider standard Han-Kobayashi (HK) techniques at both sources as well as quantize-forward (QF) and compress-forward (CF) relaying at the destinations. For the decoding techniques, we compare backward and sliding window decoding at both destinations. We prove that the achievable rate regions obtained with $Q F$ and $C F$ relaying are the same as long as the same decoding technique is used by the destinations. We also show that with backward decoding, QF and CF relaying techniques achieve the same rate region as that obtained by the HK with noisy network coding (NNC) scheme. Therefore, QF relaying is the preferred scheme because it is simpler than both $\mathrm{CF}$ relaying and NNC and still leads to the same rate region. Furthermore, we provide numerical results for the Gaussian channel. These results show that destination cooperation is more efficient in strong IC than in weak IC. Also, if the destinations have enough powers or the inter-destination links are very strong, sliding window decoding achieves almost the same rate region as backward decoding while having a much shorter decoding delay.
\end{abstract}

\section{INTRODUCTION}

The simplest interference network consists of two sourcedestination pairs. Each destination is interested in the information coming from a source and considers signals coming from the other source as interference. This interference limits the achievable rates and presents a challenging problem to designers of communication networks. Cooperation among nodes provides a valuable solution to alleviate interference and enlarge the achievability. Destination or source cooperation in the interference channel (IC) has received a heightened interest of research as in [1]-[6]. This paper concentrates on the IC with destination cooperation (IC-DC).

IC-DC is feasible in many practical applications. For example, the downlink in cellular networks present a natural scenario for the IC-DC because of the nature of wireless transmission. In the uplink of cellular networks, IC-DC is also possible via the backhaul network connecting the base stations. In [3], Prabhakaran and Viswanath propose a coding scheme based on block Markov encoding, Han-Kobayashi (HK) scheme at both sources, compress-forward (CF) relaying and backward decoding at both destinations. They characterize the sum capacity within a constant gap. In [1], Wang and Tse propose two schemes for IC with conferencing decoders both based on the HK scheme at the sources. At the destinations, the first scheme is based on CF relaying at both destinations while the other scheme is based on compress-bin-and-forward at one destination and decode-bin-and-forward at the other. They also characterize the capacity within a constant gap. In [6], Do et al. combine HK and noisy network coding (NNC) to propose another scheme for IC-DC.

In this paper, we provide a comprehensive analysis for several encoding and decoding techniques for IC-DC and compare with existing schemes. The encoding at the sources is based on the HK scheme. The destinations employs QF or $\mathrm{CF}$ relaying for encoding and backward or sliding window for decoding. We show that $\mathrm{CF}$ and $\mathrm{QF}$ relaying with backward decoding lead to the same rate region. Similar conclusion holds for sliding window decoding. QF relaying has simpler encoding than CF relaying because it does not use Wyner-Ziv binning. However, in sliding window decoding, CF relaying allows sequential decoding instead of joint decoding used in QF relaying.

We compare the resulted achievable rate regions with existing ones. For the CF-HK-based schemes in [1] and [3], we show that the proposed CF with backward decoding scheme achieves a larger region than the coding scheme in [3] and the same region as the first scheme in [1] after adapting it to the IC-DC. Furthermore, we show that backward decoding, with either $\mathrm{CF}$ or $\mathrm{QF}$ relaying techniques, leads to the same rate region achieved by the NNC scheme in [6]. We then apply the coding schemes into the Gaussian channel and illustrate numerically that destination cooperation significantly enlarges the achievable region in strong IC. In addition, the rate region achieved by sliding window decoding approaches that achieved by backward decoding as the destinations powers increase and the inter-destination link qualities improve.

\section{Channel Model}

\section{A. Discrete memoryless model}

The discrete memoryless IC-DC with two source-destination pairs $\left(\left(S_{1}, D_{1}\right),\left(S_{2}, D_{2}\right)\right)$ can be defined as shown in Figure 1. It consists of 4 input alphabets $\mathcal{X}_{1}, \mathcal{X}_{2}, \mathcal{X}_{3}$ and $\mathcal{X}_{4}$, 2 output alphabets $\mathcal{Y}_{3}$ and $\mathcal{Y}_{4}$ and is characterized by the transition probability $p\left(y_{3}, y_{4} \mid x_{1}, x_{2}, x_{3}, x_{4}\right)$. Each source has a message to send to its destination. A $\left(\left\lceil 2^{n R_{1}}\right\rceil,\left\lceil 2^{n R_{2}}\right\rceil, n\right)$ code for this channel consists of two independent message sets $W_{1}=\left\{1, \ldots,\left\lceil 2^{n R_{1}}\right\rceil\right\}$, and $W_{2}=\left\{1, \ldots,\left\lceil 2^{n R_{2}}\right\rceil\right\}$, four encoding functions $\left(f_{1 i}, f_{2 i}, f_{3 i}, f_{4 i} i=1, \ldots, n\right)$, and two decoding functions $\left(g_{1}, g_{2}\right)$ defined as

$$
f_{1 i}: W_{1} \rightarrow \mathcal{X}_{1}, \quad f_{2 i}: W_{2} \rightarrow \mathcal{X}_{2},
$$




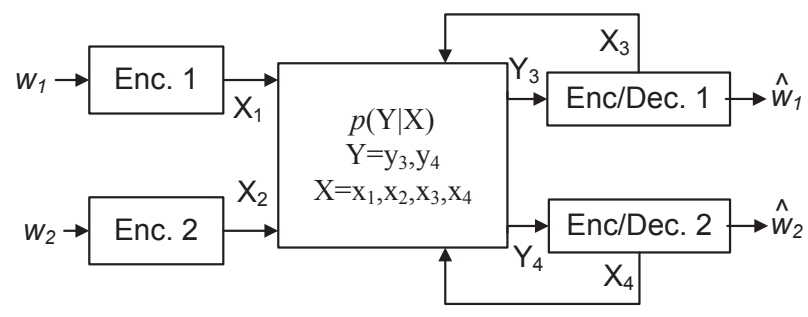

Fig. 1. The channel model for IC-DC.

$$
\begin{aligned}
& f_{3 i}: \mathcal{Y}_{3}^{i-1} \rightarrow \mathcal{X}_{3}, f_{4 i}: \mathcal{Y}_{4}^{i-1} \rightarrow \mathcal{X}_{4}, i=1, \ldots, n \\
& g_{1}: \mathcal{Y}_{3}^{n} \rightarrow \hat{W}_{1}, \quad g_{2}: \mathcal{Y}_{4}^{n} \rightarrow \hat{W}_{2} .
\end{aligned}
$$

Definitions for the average error probability, achievable rate and capacity region follow the standard ones in [7].

\section{B. Gaussian Channel Model}

The discrete-time channel model for the IC-DC over Gaussian noises can be expressed as

$$
\begin{aligned}
& Y_{3}=c_{13} X_{1}+c_{23} X_{2}+c_{43} X_{4}+Z_{3}, \\
& Y_{4}=c_{14} X_{1}+c_{24} X_{2}+c_{34} X_{3}+Z_{4},
\end{aligned}
$$

where $X_{i}$ is the transmitted signal of node $i \in\{1,2,3,4\} ; Y_{3}$ and $Y_{4}$ are the received signals at the destinations; $c_{i j}$ is the link coefficient from node $i$ to node $j$ where $i, j \in\{1,2,3,4\}$; and $Z_{3}$ and $Z_{4} \sim C N(0,1)$ are independent complex AWGN.

\section{ENCODIng TECHNIQUES}

In this section, we describe two encoding techniques for the IC-DC. These techniques are based on block Markov encoding, $\mathrm{HK}$ coding at the sources and $\mathrm{QF}$ or $\mathrm{CF}$ relaying the destinations. Consider the joint input distribution as

$$
p_{r}=p\left(x_{1}, u_{1}\right) p\left(x_{2}, u_{2}\right) p\left(\hat{y}_{3}, x_{3}\right) p\left(\hat{y}_{4}, x_{4}\right) .
$$

Then, we have two encoding techniques as follows.

\section{A. Quantize-Forward (QF) Relaying Technique}

In $\mathrm{QF}$ relaying, the relay forwards a quantized version of its received signal. This techniques is used for network coding schemes in [8] and [9]. Compared with CF relaying, this technique does not increase the achievable rate of the classical relay channel. However, it increases the achievable rate region for the TWRC as shown in [8] and [9]. Therefore, it is also of interest to study the impact of this technique on the IC-DC. Since the source-destination pairs are symmetric, we describe this encoding technique at the first pair $\left(S_{1}, D_{1}\right)$ only. In block $j, S_{1}$ splits it message into 2 parts $w_{1, j}=\left(w_{10, j}, w_{11, j}\right)$. It first generates a codeword $U_{1}$ for $w_{10, j}$ and then superimposes $w_{11, j}$ on $U_{1}$ to generate the codeword $X_{1}\left(w_{11, j}, w_{10, j}\right) . D_{1}$ generates the codewords $X_{3}\left(l_{j-1}\right)$ and $\hat{Y}_{3}\left(l_{j} \mid l_{j-1}\right)$.

1) Codebook generation: Two sequences of $B-1$ messages are sent over the channel in $n B$ transmissions. Therefore, the achievable rates are reduced by a factor of $1 / B$. However, this factor becomes negligible as $B \rightarrow \infty$. In block $j$, after fixing $p_{r}$ in (3),

- Generate $2^{n R_{10}}$ i.i.d sequences $u_{1}^{n}\left(w_{10, j}\right) \sim$ $\prod_{k=1}^{n} p\left(u_{1 k}\right)$.
- For each $u_{1}^{n}$, generate $2^{n R_{11}}$ i.i.d sequences $x_{1}^{n}\left(w_{11, j}, w_{10, j}\right) \sim \prod_{k=1}^{n} p\left(x_{1 k} \mid u_{1 k}\right)$.

- Generate $2^{n R_{3}}$ i.i.d sequences $x_{3}^{n}\left(l_{3, j-1}\right) \sim$ $\prod_{k=1}^{n} p\left(x_{3 k}\right)$.

- For each $x_{3}^{n}$, generate $2^{n R_{3}}$ i.i.d sequences $\hat{y}_{3}^{n}\left(l_{3, j}, l_{3, j-1}\right) \sim \prod_{k=1}^{n} p\left(\hat{y}_{3 k} \mid x_{3 k}\right)$.

- Similarly generate $u_{2}^{n}\left(w_{20, j}\right), \quad x_{2}^{n}\left(w_{21, j}, w_{20, j}\right)$, $x_{4}^{n}\left(l_{4, j-1}\right)$ and $\hat{y}_{4}^{n}\left(l_{4, j}, l_{4, j-1}\right)$.

2) Encoding: In block $j$, let $\left(w_{1, j}, w_{2, j}\right)$ be the new messages to be sent. Then, $S_{1}$ and $S_{2}$ simply send $x_{1}^{n}\left(w_{11, j}, w_{10, j}\right)$ and $x_{2}^{n}\left(w_{21, j}, w_{20, j}\right)$, respectively. Besides, $D_{1}$ knows $L_{3, j-1}$ and it quantizes $Y_{3}^{n}(j)$ by finding an index $\hat{l}_{3, j}$ such that

$$
\left(\hat{Y}_{3}\left(\hat{l}_{3, j} \mid L_{3, j-1}\right), X_{3}\left(L_{3, j-1}\right), Y_{3}^{n}(j)\right) \in A_{\epsilon}^{n}
$$

By the covering lemma, such $\hat{l}_{3, j}$ exists if

$$
R_{3}>I\left(\hat{Y}_{3} ; Y_{3} \mid X_{3}\right)
$$

Similar decoding at $D_{2}$ leads to $R_{4}>I\left(\hat{Y}_{4} ; Y_{4} \mid X_{4}\right)$. Then, $D_{1}$ and $D_{2}$ send $x_{3}^{n}\left(l_{3, j}\right)$ and $x_{4}^{n}\left(l_{4, j}\right)$ in block $j+1$, respectively.

\section{B. Compress-Forward (CF) Relaying Technique}

A CF encoding technique for IC-DC is proposed in [3]. However, in this section, we propose another CF encoding technique that includes the proposed in [3] as a special case. Detailed comparison is given in Section VI.

1) Codebook generation: The codebook generation for the $\mathrm{CF}$ encoding technique is similar to the $\mathrm{QF}$ technique where after fixing $p_{r}$ in (3), generate $u_{1}^{n}\left(w_{10, j}\right), u_{2}^{n}\left(w_{20, j}\right)$, $x_{1}^{n}\left(w_{11, j}, w_{10, j}\right), x_{2}^{n}\left(w_{21, j}, w_{20, j}\right), x_{3}^{n}\left(l_{3, j-1}\right)$, and $x_{4}^{n}\left(l_{4, j-1}\right)$ exactly as in QF technique. Then,

- for each $x_{3}^{n}\left(l_{3, j-1}\right)$, generate $2^{n \tilde{R}_{3}}$ i.i.d sequences $\hat{y}_{3}^{n}\left(k_{3, j}, l_{3, j-1}\right) \sim \prod_{i=1}^{n} p\left(\hat{y}_{3 i} \mid x_{3 i}\right) . l_{3, j}$ and $k_{3, j}$ are the binning and compression indices, respectively for the received signal $Y_{3}$ at $D_{1}$.

- similarly generate $\hat{y}_{4}^{n}\left(k_{4, j}, l_{4, j-1}\right)$.

2) Encoding: The encoding scheme is similar to that of the QF technique except that $D_{1}$ finds $\hat{k}_{3, j}$ instead of $\hat{l}_{3, j}$ in (4). Therefore, we obtain a similar constraint as in (5) but replacing $R_{3}$ by $\tilde{R}_{3}$. Similarly, $\tilde{R}_{4}>I\left(\hat{Y}_{4} ; Y_{4} \mid X_{4}\right)$.

\section{Decoding TeChniQues}

In this section, we consider two decoding techniques: backward and sliding window decoding. Although backward decoding usually enlarges the achievable rate region, it leads to a long delay. Therefore, sliding window decoding can provide a suitable solution for delay sensitive applications.

\section{A. Backward Decoding}

In backward decoding, the destinations start decoding after the end of the last transmission block. For QF encoding technique, $D_{1}$ knows $L_{4, j}$ in block $j$ and looks for a unique $\left(\hat{w}_{10, j}, \hat{w}_{11, j}, \hat{l}_{4, j-1}\right)$ for some $\hat{w}_{20, j}$ such that

$U_{1}\left(\hat{w}_{10, j}\right), X_{1}\left(\hat{w}_{11, j}, \hat{w}_{10, j}\right), U_{2}\left(\hat{w}_{20, j}\right), \hat{Y}_{4}\left(L_{4, j} \mid \tilde{l}_{4, j-1}\right)$, 
For the CF relaying technique, $D_{1}$ knows $L_{4, j}$ and looks for a unique message vector $\left(\hat{w}_{10, j}, \hat{w}_{11, j}, \hat{l}_{4, j-1}\right)$ for some $\hat{w}_{20, j}$ and $\hat{k}_{4, j} \in L_{4, j}$ such that the same decoding rule in (6) is satisfied but after replacing $L_{3, j}$ by $K_{3, j}$ and $L_{4, j}$ by $\hat{k}_{4, j}$.

\section{B. Sliding Window Decoding}

In sliding window decoding, the destinations decode the messages sent in block $j$ after receiving the signals in blocks $j$ and $j+1$. Hence, this decoding has only one block delay.

1) For $Q F$ Encoding Technique: $D_{1}$ utilizes $Y_{3}(j)$ and $Y_{3}(j+1)$ and perform joint decoding by looking for a unique $\left(\hat{w}_{10, j}, \hat{w}_{11, j}, \hat{l}_{4, j}\right)$ for some $\hat{w}_{20, j}$ such that

$$
\begin{array}{r}
\left(U_{1}\left(\hat{w}_{10, j}\right), X_{1}\left(\hat{w}_{11, j}, \hat{w}_{10, j}\right), U_{2}\left(\hat{w}_{20, j}\right), \hat{Y}_{4}\left(\hat{l}_{4, j} \mid L_{4, j-1}\right),\right. \\
\left.X_{4}\left(L_{4, j-1}\right), \hat{Y}_{3}\left(L_{3, j} \mid L_{3, j-1}\right), X_{3}\left(L_{3, j-1}\right), Y_{3}(j)\right) \in A_{\epsilon}^{n} \\
\text { and }\left(X_{4}\left(\hat{l}_{4, j}\right), \hat{Y}_{3}\left(L_{3, j+1} \mid L_{3, j}\right), X_{3}\left(L_{3, j}\right), Y_{3}(j+1)\right) \in A_{\epsilon}^{n}
\end{array}
$$

2) For CF Encoding Technique: $D_{1}$ decodes the binning index $\left(\hat{l}_{4, j}\right)$ in block $j+1$ from the received signal $Y_{3}(j+1)$ and then goes back to block $j$ to decode the message parts for some compression index $\hat{k}_{4, j}$. Hence, in block $j+1, D_{1}$ looks for a unique $\hat{l}_{4, j}$ such that

$$
\left(X_{4}\left(\hat{l}_{4, j}\right), \hat{Y}_{3}\left(K_{3, j+1} \mid L_{3, j}\right), X_{3}\left(L_{3, j}\right), Y_{3}(j+1)\right) \in A_{\epsilon}^{n}
$$

The error analysis leads to the rate constraint $R_{4} \leq$ $I\left(X_{4} ; Y_{3} \mid X_{3}\right)$. Similar decoding at $D_{2}$ leads to $R_{3} \leq$ $I\left(X_{3} ; Y_{4} \mid X_{4}\right)$. Then, $D_{1}$ looks for a unique $\left(\hat{w}_{10, j}, \hat{w}_{11, j}\right)$ and some $\hat{w}_{20, j}$, and $\tilde{k}_{4, j} \in L_{4, j}$ such that

$$
\begin{array}{r}
\left(U_{1}\left(\hat{w}_{10, j}\right), X_{1}\left(\hat{w}_{11, j}, \hat{w}_{10, j}\right), U_{2}\left(\hat{w}_{20, j}\right) \hat{Y}_{4}\left(\tilde{k}_{4, j} \mid L_{4, j-1}\right)\right. \\
\left.X_{4}\left(L_{4, j-1}\right), \hat{Y}_{3}\left(K_{3, j} \mid L_{3, j-1}\right), X_{3}\left(L_{3, j-1}\right), Y_{3}(j)\right) \in A_{\epsilon}^{n}
\end{array}
$$

\section{Achievable Rate Regions}

In order to analyze the different encoding and decoding techniques, we compare between the rate regions achieved by these techniques. Define the following parameters:

$$
\begin{aligned}
\mu_{0} & =I\left(\hat{Y}_{4} ; U_{2}, X_{1}, X_{3}, Y_{3} \mid X_{4}\right), \\
\tilde{\mu}_{0} & =I\left(U_{1}, X_{2}, X_{4}, Y_{4} ; \hat{Y}_{3} \mid X_{3}\right), \\
\mu_{1} & =I\left(\hat{Y}_{3} ; Y_{3} \mid X_{3}\right), \mu_{2}=I\left(\hat{Y}_{4} ; Y_{4} \mid X_{4}\right), \\
\mu_{3} & =I\left(X_{1} ; \hat{Y}_{4}, Y_{3} \mid U_{1}, U_{2}, X_{3}, X_{4}\right), \\
\mu_{4} & =I\left(X_{1} ; \hat{Y}_{4}, Y_{3} \mid U_{2}, X_{3}, X_{4}\right), \\
\mu_{5} & =I\left(U_{2}, X_{1} ; \hat{Y}_{4}, Y_{3} \mid U_{1}, X_{3}, X_{4}\right), \\
\mu_{6} & =I\left(U_{2}, X_{1} ; \hat{Y}_{4}, Y_{3} \mid X_{3}, X_{4}\right), \\
\mu_{7} & =I\left(X_{1} ; Y_{3} \mid U_{1}, U_{2}, X_{3}, X_{4}\right)+\mu_{0}, \\
\mu_{8} & =I\left(X_{1} ; Y_{3} \mid U_{2}, X_{3}, X_{4}\right)+\mu_{0}, \\
\mu_{9} & =I\left(U_{2}, X_{1} ; Y_{3} \mid U_{1}, X_{3}, X_{4}\right)+\mu_{0}, \\
\mu_{10} & =I\left(U_{2}, X_{1} ; Y_{3} \mid X_{3}, X_{4}\right)+\mu_{0}, \\
\mu_{11} & =\mu_{7}+I\left(X_{4} ; Y_{3} \mid U_{1}, U_{2}, X_{3}\right), \\
\mu_{12} & =\mu_{8}+I\left(X_{4} ; Y_{3} \mid U_{2}, X_{3}\right), \\
\mu_{13} & =\mu_{9}+I\left(X_{4} ; Y_{3} \mid U_{1}, X_{3}\right), \\
\mu_{14} & =\mu_{10}+I\left(X_{4} ; Y_{3} \mid X_{3}\right),
\end{aligned}
$$

Then, we have the following Theorems:
Theorem 1. The rate region for the IC-DC obtained by $Q F$ encoding with backward decoding techniques is the convex closure of the rate-tuples $\left(R_{10}, R_{11}, R_{20}, R_{21}\right)$ satisfying

$$
\begin{aligned}
R_{11} & \leq \min \left\{\mu_{3}, \mu_{11}-\mu_{2}\right\}=A_{1}, \\
R_{1} & \leq \min \left\{\mu_{4}, \mu_{12}-\mu_{2}\right\}=A_{2}, \\
R_{11}+R_{20} & \leq \min \left\{\mu_{5}, \mu_{13}-\mu_{2}\right\}=A_{3}, \\
R_{1}+R_{20} & \leq \min \left\{\mu_{6}, \mu_{14}-\mu_{2}\right\}=A_{4},
\end{aligned}
$$

and other 4 constraints $A_{5}-A_{8}$ similar to $A_{1}, \ldots, A_{4}, o b$ tained by switching all the first indices from $1 \rightarrow 2$ and $2 \rightarrow 1$, and replacing $Y_{3}$ with $Y_{4}$. These constraints hold for some joint distribution $p_{r}$ as in (3) and are subject to

$$
\begin{aligned}
& I\left(\hat{Y}_{3} ; Y_{3} \mid X_{3}\right) \leq \tilde{\mu}_{0}+I\left(X_{3} ; Y_{4} \mid X_{2}, U_{1}, X_{4}\right), \\
& I\left(\hat{Y}_{4} ; Y_{4} \mid X_{4}\right) \leq \mu_{0}+I\left(X_{4} ; Y_{3} \mid X_{1}, U_{2}, X_{3}\right) .
\end{aligned}
$$

Proof: The error analysis or the decoding rule in (6) leads to the following constraints:

$$
\begin{array}{rlrl}
R_{4} & \leq I\left(X_{4} ; Y_{3} \mid X_{1}, U_{2}, X_{3}\right)+\mu_{0}, \\
R_{11} & \leq \mu_{3}, & R_{1} \leq \mu_{4}, \\
R_{11}+R_{20} & \leq \mu_{5}, & R_{1}+R_{20} \leq \mu_{6}, \\
R_{11}+R_{4} & \leq \mu_{11}, & R_{1}+R_{4} \leq \mu_{12}, \\
R_{11}+R_{20}+R_{4} & \leq \mu_{13}, & R_{1}+R_{20}+R_{4} \leq \mu_{14},
\end{array}
$$

Then, the rate region is obtained by combining (5) and (13).

Theorem 2. The rate region for the $I C-D C$ obtained by $C F$ with backward decoding is identical to that in Theorem 1. Therefore, with backward decoding, $C F$ and $Q F$ are equivalent in terms of the achievable regions.

Proof: Error analysis of the decoding rule for $\mathrm{CF}$ with backward decoding leads to the following constraints:

$$
\begin{aligned}
R_{4} & \leq I\left(X_{4} ; Y_{3} \mid X_{1}, U_{2}, X_{3}\right), \\
R_{11}+\tilde{R}_{4}-R_{4} & \leq \mu_{7}, \\
R_{1}+\tilde{R}_{4}-R_{4} & \leq \mu_{8}, \\
R_{11}+R_{20}+\tilde{R}_{4}-R_{4} & \leq \mu_{9}, \\
R_{1}+R_{20}+\tilde{R}_{4}-R_{4} & \leq \mu_{10},
\end{aligned}
$$

where the first constraint is obtained from the decoding in the last block. Then, an achievable rate region identical to that in Theorem 1 is obtained by combining $\tilde{R}_{3}>\mu_{1}$ and $\tilde{R}_{4}>\mu_{2}$ with the rate constraints stemmed from the decoding at the destinations.

Theorem 3. The rate region for the IC-DC obtained by $Q F$ with sliding window decoding is similar to that with backward decoding but replacing $I\left(X_{4} ; Y_{3} \mid U_{1}, U_{2}, X_{3}\right), \quad I\left(X_{4} ; Y_{3} \mid U_{2}, X_{3}\right)$ and $I\left(X_{4} ; Y_{3} \mid U_{1}, X_{3}\right)$ in $\mu_{11}, \mu_{12}$, and $\mu_{13}$ all by $I\left(X_{4} ; Y_{3} \mid X_{3}\right)$, and replacing (12) by

$$
\begin{aligned}
& I\left(\hat{Y}_{3} ; Y_{3} \mid X_{3}\right) \leq \tilde{\mu}_{0}+I\left(X_{3} ; Y_{4} \mid X_{4}\right), \\
& I\left(\hat{Y}_{4} ; Y_{4} \mid X_{4}\right) \leq \mu_{0}+I\left(X_{4} ; Y_{3} \mid X_{3}\right) .
\end{aligned}
$$


Proof: The theorem is obtained from combining (5) and the rate constraints stemmed from the decoding rule in (7).

Theorem 4. The rate region for the IC-DC obtained by $C F$ with sliding window decoding is similar to that obtained by the QF scheme given in Theorem 3 but without the conditions in (15). The two rate regions are equivalent despite condition (15) in Theorem 3.

Proof: The theorem is obtained from combining $\tilde{R}_{3}>\mu_{1}$ and $\tilde{R}_{4}>\mu_{2}$ and the rate constraints stemmed from the joint typicality rules in (8) and (9). Although this region seems larger than that in Theorem 3 because of the absence of the conditions in (15), these two conditions are redundant as shown in Appendix A.

\section{COMPARISON WITH EXISTING SCHEMES}

In this section, we compare our $\mathrm{QF}$ and $\mathrm{CF}$ schemes with existing schemes in [1], [3] and [6], respectively.

\section{A. I. Wang and D. Tse [1]}

Two schemes are proposed for the IC with conferencing decoders. Both scheme employs HK coding at each source. At the destinations, the first scheme employs $\mathrm{CF}$ relaying. The second scheme employs a two-step decoding technique which can be described as follows.

- $D_{2}$ employs CF of its received signal.

- $D_{1}$ correctly decodes both public message parts and the private part $w_{11}$ for some bin index sent by $D_{2}$.

- $D_{1}$ bins each of the public message part and sends the bin indices to $D_{2}$.

- $D_{2}$ decodes $\left(w_{11}, w_{21}\right)$ for some $w_{10}$ and bin indices.

The achievable region in Theorem 4.3 in [1] is obtained by the union of the two achievable regions resulting from this decoding order and the opposite order.

Remark 1. Adapting the first scheme of the IC with conferencing decoders into the IC-DC and employing backward decoding leads to the same rate region as CF with backward decoding in Theorem 2.

\section{B. V. Prabhakaran and P. Viswanath [3]}

Prabhakaran and Viswanath propose a scheme for IC-DC based on HK coding at both sources and CF relaying and backward decoding at both destinations. Hence, this scheme appears to be similar to our $\mathrm{CF}$ and backward decoding scheme. However, there are some important differences in this scheme as follows:

- Each destination needs to correctly decode the public parts of both sources which leads to additional constraints in the rate region.

- The codewords for the compression and binning indices are independent. However, they are usually dependent. Making them independent increases the rate constraints and hence reduces the rate region.

Remark 2. The proposed scheme in [3] is a special case of the $\mathrm{CF}$ with backward decoding scheme. This can be verified by generating independent codewords for the compression and binning indices and by not requiring each destination to correctly decode the public message part of the undesired source.

\section{H. Do, T. Oechtering and M. Skoglun [6]}

In [6], Do et al. combine the HK and NNC schemes to propose a coding scheme for the IC-DC. In this scheme, each source employs HK coding, each destination employs $\mathrm{QF}$ relaying, and the same message is sent over $B$ blocks. Then, at the end of the last transmission block, each destination employs simultaneous joint decoding over all transmission blocks.

The probabilities of error events in [6] are bounded in two ways following the same procedure in [1]. However, if we only consider the standard one as used in [8], we obtain the following Theorem:

Theorem 5. The achievable rate region for the IC-DC obtained by the HK and NNC scheme in [6] is the same as by $Q F$ or $C F$ with backward decoding in Theorem 1 or 2 but without the conditions in (12). The three rate regions are equivalent despite conditions (12) in Theorems 1 and 2.

Proof: Appendix B shows that the rate constraints stemmed from the $\mathrm{HK}$ and $\mathrm{NNC}$ scheme in [6] are identical to those in Theorem 1. CF and QF with backward decoding schemes are equivalent as shown in Theorem 2. Moreover, following similar approach for the proof of Theorem 4, we can show that the conditions in (12) are redundant. Hence, CF, QF with backward decoding and NNC schemes are equivalent. The detailed proof is removed because of space limitation.

\section{RESUlts FOR THE GAUSSIAN CHANNEL}

The discrete-time model for the Gaussian IC-DC is given in (2). The signalings for the $\mathrm{CF}$ and $\mathrm{QF}$ schemes are identical for Gaussian channels. The signaling for the first sourcedestination pair $\left(S_{1}, D_{1}\right)$ in each transmission block is given as follows.

$$
\begin{aligned}
& X_{1}=\sqrt{\rho_{11}} T_{1}\left(w_{11, j}\right)+\sqrt{\rho_{10}} U_{1}\left(w_{10, j}\right), \\
& X_{3}=\sqrt{P_{3}} T_{3}\left(l_{j-1}\right), \quad \hat{Y}_{3}=Y_{3}+\hat{Z}_{3},
\end{aligned}
$$

where $\hat{Z}_{3} \sim \mathcal{C N}\left(0, \sigma^{2}\right)$ and $T_{1}, T_{3}$ and $U_{1}$ are all i.i.d random variables distributed according to $\sim \mathcal{C N}(0,1)$. The power constraint at $S_{1}$ is given as $\rho_{11}+\rho_{10}=P_{1}$. Similar signaling holds for the other source-destination pair $\left(S_{2}, D_{2}\right)$. We remove the achievable rate regions because of space limitation. However, they can be derived easily by applying Theorems 1, 3, 2 and 4 to the Gaussian channel in (2) with the signaling in (16).

Figures 2 and 3 present some numerical results for the IC-DC for strong and weak interference cases, respectively. These results show that destination cooperation effectively enlarges the achievable region in strong interference while only slightly enlarges it in weak interference. Moreover, as the destinations powers $\left(P_{3}\right.$ and $\left.P_{4}\right)$ increase and/or the inter-destination links $\left(c_{34}, c_{43}\right)$ improve, rate regions for sliding window decoding schemes become closer to those of backward decoding schemes. For practical designers, using sliding window decoding is preferred in these cases because it 


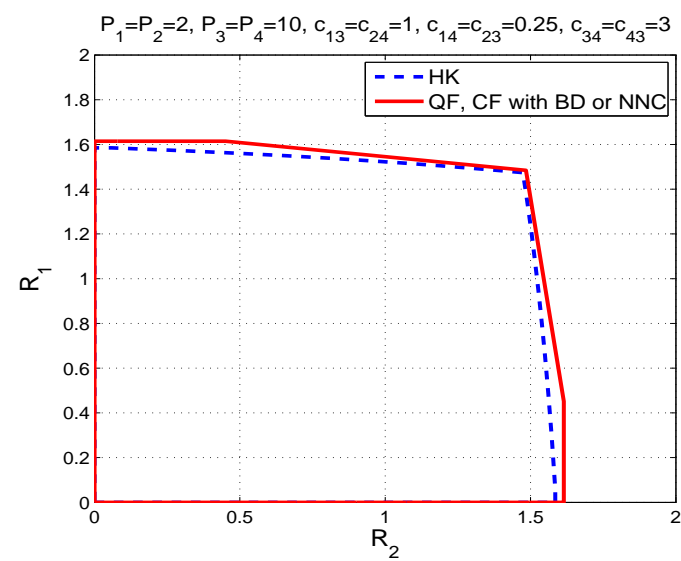

Fig. 2. Comparison between different IC-DC schemes and the HK region in weak interference (BD: backward decoding).

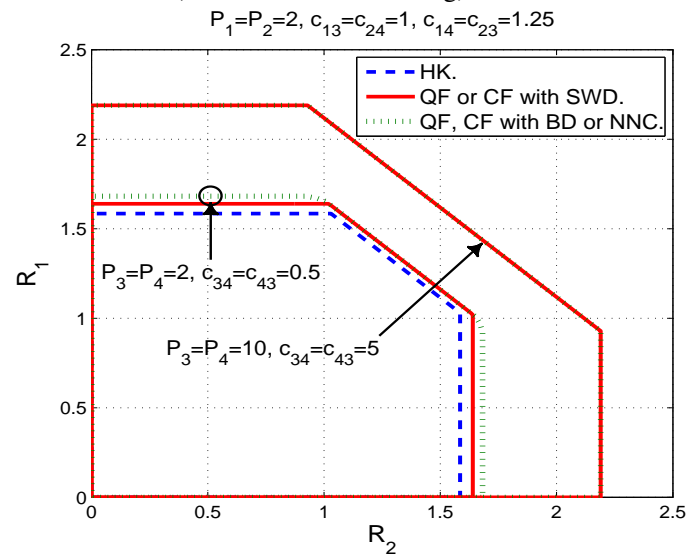

Fig. 3. Comparison between different IC-DC schemes and the HK region in strong interference (SWD: sliding window decoding).

can achieve almost the same rate region as backward decoding but with a much shorter decoding delay.

\section{CONCLUSION}

We have analyzed different encoding and decoding techniques for the IC-DC. We use the HK technique at the sources, QF or CF relaying and sliding window or backward decoding techniques at the destinations. We show that $\mathrm{CF}$ or QF with backward decoding leads to the same rate regions as the NNC scheme. Moreover, we show that $\mathrm{CF}$ and QF with sliding window decoding are equivalent. We also present numerical results for Gaussian channels. Drawing on these results, destination cooperation is more efficient in strong interference than in weak interference. In addition, sliding window decoding is preferred when the inter-destination links are very strong and/or when the destinations have enough powers.

\section{APPENDIX A \\ PROOF OF THEOREM 4}

In this section, we show that the achievable region in Theorem 4 obtained by CF or QF with sliding window decoding are equivalent. Simply, we need to show that the constraints in (15) are redundant. This can be done by following similar procedure used in [9]-[11].

First, lets define the following Lemma:
Lemma 1. The achievable rate region given in Theorem 4 for $C F$ with sliding window decoding can be alternatively expressed as

$$
\begin{aligned}
R_{11} & \leq \mu_{3}, \quad R_{1} \leq \mu_{4}, \quad R_{21} \leq \mu_{15}, \quad R_{2} \leq \mu_{16} \\
R_{11}+R_{20} & \leq \mu_{5}, \quad R_{1}+R_{20} \leq \mu_{6} \\
R_{21}+R_{10} & \leq \mu_{17}, \quad R_{2}+R_{10} \leq \mu_{18}
\end{aligned}
$$

and these constraints are subject to

$$
\begin{aligned}
& I\left(X_{3} ; Y_{4} \mid X_{4}\right) \geq I\left(\hat{Y}_{3} ; Y_{3} \mid X_{3}, X_{4}, Y_{4}\right), \\
& I\left(X_{4} ; Y_{3} \mid X_{3}\right) \geq I\left(\hat{Y}_{4} ; Y_{4} \mid X_{3}, X_{4}, Y_{3}\right) .
\end{aligned}
$$

Proof:

- Achievability:

Following similar encoding and decoding for the proposed scheme in (11) but after decoding $\hat{l}_{2, j}$ using the decoding rule in (8), $D_{1}$ looks for $\hat{k}_{2, j}$ such that

$$
\begin{aligned}
& \left(\hat{Y}_{4}\left(\hat{k}_{2, j} \mid L_{j-1}\right), \hat{Y}_{3}\left(K_{1, j} \mid L_{1, j-1}\right),\right. \\
& \left.\quad X_{4}\left(L_{2, j-1}\right), X_{3}\left(L_{1, j-1}\right), Y_{3}(j)\right) \in A_{\epsilon}^{n}
\end{aligned}
$$

Error analysis leads to the constraint

$$
\tilde{R}_{4}-R_{4} \leq I\left(\hat{Y}_{4} ; X_{3}, Y_{3} \mid X_{4}\right)
$$

By combining (20), $\mu_{1}$ in (10) and $R_{4} \leq I\left(X_{4} ; Y_{3} \mid X_{3}\right)$, we obtain the condition

$$
\begin{aligned}
I\left(X_{4} ; Y_{3} \mid X_{3}\right) & \geq I\left(\hat{Y}_{4} ; Y_{4} \mid X_{4}\right)-I\left(\hat{Y}_{3} ; X_{3}, Y_{3} \mid X_{4}\right), \\
& =I\left(\hat{Y}_{4} ; X_{3}, Y_{3}, Y_{4} \mid X_{4}\right)-I\left(\hat{Y}_{3} ; X_{3}, Y_{3} \mid X_{4}\right) \\
& =I\left(\hat{Y}_{4} ; Y_{4} \mid X_{3}, X_{4}, Y_{3}\right) .
\end{aligned}
$$

Similarly, from the decoding at $D_{2}$, we obtain the condition

$$
I\left(X_{3} ; Y_{4} \mid X_{4}\right) \geq I\left(\hat{Y}_{3} ; Y_{3} \mid X_{3}, X_{4}, Y_{4}\right) .
$$

Next, $D_{1}$ looks for a unique $\left(\hat{w}_{10, j}, \hat{w}_{11, j}\right)$ and some $\hat{w}_{20, j}$ such that

$$
\begin{aligned}
& \left(U_{1}\left(\hat{w}_{10, j}\right), X_{1}\left(\hat{w}_{11, j}, \hat{w}_{10, j}\right), U_{2}\left(\hat{w}_{20, j}\right), X_{4}\left(L_{2, j-1}\right),\right. \\
& \left.\hat{Y}_{4}\left(K_{2, j} \mid L_{j-1}\right), \hat{Y}_{3}\left(K_{1, j} \mid L_{1, j-1}\right), X_{3}\left(L_{1, j-1}\right), Y_{3}(j)\right) \in A_{\epsilon}^{n}
\end{aligned}
$$

Error analysis leads to the first 4 rate constraints in (17). The other constraints are similarly obtained from the decoding at $D_{2}$

- Converse:

Here, we prove that any rate tuples satisfying (17) and the conditions in (18) also satisfy (11). Because of similar analysis, we prove the converse for $R_{11}$ only.

$$
\begin{aligned}
& R_{11} \leq \mu_{3}=I\left(X_{1} ; Y_{3}, \hat{Y}_{4} \mid U_{1}, U_{2}, X_{3}, X_{4}\right), \\
& =H\left(Y_{3}, \hat{Y}_{4} \mid U_{1}, U_{2}, X_{3}, X_{4}\right)-H\left(Y_{3}, \hat{Y}_{4} \mid U_{1}, U_{2}, X_{1}, X_{3}, X_{4}\right), \\
& =H\left(Y_{3} \mid U_{1}, U_{2}, X_{3}, X_{4}\right)+H\left(\hat{Y}_{4} \mid Y_{3}, U_{1}, U_{2}, X_{3}, X_{4}\right) \\
& \quad-H\left(\hat{Y}_{4} \mid U_{1}, U_{2}, X_{1}, X_{3}, X_{4}\right) \\
& \quad-H\left(Y_{3} \mid \hat{Y}_{4}, U_{1}, U_{2}, X_{1}, X_{3}, X_{4}\right),
\end{aligned}
$$




$$
\begin{aligned}
& =-I\left(U_{1}, U_{2} ; Y_{3} \mid X_{3}, X_{4}\right)+H\left(Y_{3} \mid X_{3}, X_{4}\right) \\
& -I\left(U_{1}, U_{2} ; \hat{Y}_{4} \mid Y_{3}, X_{3}, X_{4}\right)-H\left(\hat{Y}_{4} \mid Y_{4}, Y_{3}, X_{3}, X_{4}\right) \\
& \text { - } H\left(\hat{Y}_{4} \mid U_{1}, U_{2}, X_{1}, X_{3}, X_{4}\right) \\
& \text { - } H\left(Y_{3} \mid \hat{Y}_{4}, U_{1}, U_{2}, X_{1}, X_{3}, X_{4}\right) \text {, } \\
& =-I\left(U_{1}, U_{2} ; Y_{3}, \hat{Y}_{4} \mid X_{3}, X_{4}\right)+H\left(Y_{3} \mid X_{3}\right) \\
& +H\left(\hat{Y}_{4} \mid Y_{4}, Y_{3}, X_{3}, X_{4}\right)-I\left(X_{4}, Y_{3} \mid X_{3}\right) \\
& +I\left(Y_{4}, \hat{Y}_{4} \mid X_{3}, X_{4}, Y_{3}\right)-H\left(\hat{Y}_{4} \mid U_{1}, U_{2}, X_{1}, X_{3}, X_{4}\right) \\
& -H\left(Y_{3} \mid \hat{Y}_{4}, U_{1}, U_{2}, X_{1}, X_{3}, X_{4}\right) \text {, } \\
& \stackrel{a}{\leq}-I\left(U_{1}, U_{2} ; Y_{3}, \hat{Y}_{4} \mid X_{3}, X_{4}\right)+H\left(Y_{3} \mid X_{3}\right) \\
& +H\left(\hat{Y}_{4} \mid Y_{4}, Y_{3}, X_{3}, X_{4}\right)-H\left(\hat{Y}_{4} \mid U_{1}, U_{2}, X_{1}, X_{3}, X_{4}\right) \\
& \text { - } H\left(Y_{3} \mid \hat{Y}_{4}, U_{1}, U_{2}, X_{1}, X_{3}, X_{4}\right) \text {, } \\
& =-I\left(U_{1}, U_{2} ; Y_{3}, \hat{Y}_{4} \mid X_{3}, X_{4}\right)+H\left(Y_{3} \mid X_{3}\right) \\
& +H\left(\hat{Y}_{4} \mid Y_{4}, Y_{3}, X_{3}, X_{4}, X_{1}, U_{1}, U_{2}\right) \\
& -H\left(\hat{Y}_{4} \mid U_{1}, U_{2}, X_{1}, X_{3}, X_{4}\right) \\
& \text { - } H\left(Y_{3} \mid \hat{Y}_{4}, U_{1}, U_{2}, X_{1}, X_{3}, X_{4}\right), \\
& =-I\left(U_{1}, U_{2} ; Y_{3}, \hat{Y}_{4} \mid X_{3}, X_{4}\right)+I\left(Y_{3} ; U_{1}, U_{2}, X_{1}, X_{4}, \hat{Y}_{4} \mid X_{3}\right) \\
& -I\left(\hat{Y}_{4} ; Y_{3}, Y_{4} \mid U_{1}, U_{2}, X_{1}, X_{3}, X_{4}\right) \\
& =-I\left(U_{1}, U_{2} ; Y_{3}, \hat{Y}_{4} \mid X_{3}, X_{4}\right) \\
& +I\left(Y_{3} ; U_{1}, U_{2}, X_{1}, X_{4}, \hat{Y}_{4} \mid X_{3}\right) \\
& -I\left(\hat{Y}_{4} ; Y_{3} \mid U_{1}, U_{2}, X_{1}, X_{3}, X_{4}\right) \\
& -I\left(\hat{Y}_{4} ; Y_{4} \mid Y_{3}, U_{1}, U_{2}, X_{1}, X_{3}, X_{4}\right) \\
& =-I\left(U_{1}, U_{2} ; Y_{3} \mid X_{3}, X_{4}\right)-I\left(U_{1}, U_{2} ; \hat{Y}_{4} \mid Y_{3}, X_{3}, X_{4}\right) \\
& +I\left(X_{4} ; Y_{3} \mid X_{3}\right)+I\left(U_{1}, U_{2} ; Y_{3} \mid X_{3}, X_{4}\right) \\
& +I\left(X_{1}, \hat{Y}_{4} ; Y_{3} \mid U_{1}, U_{2}, X_{3}, X_{4}\right) \\
& -I\left(\hat{Y}_{4} ; Y_{3} \mid U_{1}, U_{2}, X_{1}, X_{3}, X_{4}\right) \\
& -I\left(\hat{Y}_{4} ; Y_{4} \mid Y_{3}, U_{1}, U_{2}, X_{1}, X_{3}, X_{4}\right) \\
& =-I\left(U_{1}, U_{2} ; \hat{Y}_{4} \mid Y_{3}, X_{3}, X_{4}\right)+I\left(X_{4} ; Y_{3} \mid X_{3}\right) \\
& +I\left(X_{1} ; Y_{3} \mid U_{1}, U_{2}, X_{3}, X_{4}\right) \\
& -I\left(\hat{Y}_{4} ; Y_{4} \mid Y_{3}, U_{1}, U_{2}, X_{1}, X_{3}, X_{4}\right) \\
& \leq I\left(X_{4} ; Y_{3} \mid X_{3}\right)+I\left(X_{1} ; Y_{3} \mid U_{1}, U_{2}, X_{3}, X_{4}\right) \\
& -I\left(\hat{Y}_{4} ; Y_{4} \mid Y_{3}, U_{1}, U_{2}, X_{1}, X_{3}, X_{4}\right)=\mu_{11}-\mu_{2} .
\end{aligned}
$$

where $(a)$ follows from the condition in (18).

Now, after proving the Lemma, in order to show that the conditions in (12) are redundant, we need to show that

$$
\begin{aligned}
& I\left(\hat{Y}_{3} ; Y_{3} \mid X_{3}\right)-\tilde{\mu}_{0} \leq I\left(X_{3} ; Y_{4} \mid X_{4}\right), \\
& I\left(\hat{Y}_{4} ; Y_{4} \mid X_{4}\right)-\mu_{0} \leq I\left(X_{4} ; Y_{3} \mid X_{3}\right) .
\end{aligned}
$$

Consider the second condition in (23) and replace $I\left(X_{4} ; Y_{3} \mid X_{3}\right)$ by $I\left(Y_{4} ; \hat{Y}_{4} \mid X_{3}, X_{4}, Y_{3}\right)$. Then, note that

$$
\begin{aligned}
& I\left(Y_{4} ; \hat{Y}_{4} \mid X_{3}, X_{4}, Y_{3}\right) \\
& =I\left(U_{1}, U_{2}, X_{1}, Y_{4} ; \hat{Y}_{4} \mid X_{3}, X_{4}, Y_{3}\right) \\
& =I\left(U_{1}, U_{2}, X_{1} ; \hat{Y}_{4} \mid X_{3}, X_{4}, Y_{3}\right)+I\left(Y_{4} ; \hat{Y}_{4} \mid U_{1}, U_{2}, X_{1}, X_{3}, X_{4}, Y_{3}\right) \\
& \geq I\left(Y_{4} ; \hat{Y}_{4} \mid U_{1}, U_{2}, X_{1}, X_{3}, X_{4}, Y_{3}\right)
\end{aligned}
$$

while the LHS of (23) can be expressed as

$$
\begin{aligned}
& I\left(\hat{Y}_{4} ; Y_{4} \mid X_{4}\right)-I\left(U_{1}, U_{2}, X_{1}, X_{3}, Y_{3} ; \hat{Y}_{4} \mid X_{4}\right) \\
& =I\left(\hat{Y}_{4} ; U_{1}, U_{2}, X_{1}, X_{3}, Y_{3}, Y_{4} \mid X_{4}\right)-I\left(U_{1}, U_{2}, X_{1}, X_{3}, Y_{3} ; \hat{Y}_{4} \mid X_{4}\right) \\
& =I\left(\hat{Y}_{4} ; Y_{4} \mid U_{1}, U_{2}, X_{1}, X_{3}, X_{4}, Y_{3}\right) .
\end{aligned}
$$

From (24) and (25), we conclude that the additional conditions in (12) are redundant. Hence, the two achievable regions for the IC-DC are equivalent.

\section{APPENDIX B \\ Proof OF THEOREM 5}

In [6], consider formula (2) in Theorem 1 . With standard error analysis used in [8], we have

$$
\begin{aligned}
R_{1} & \leq \min \left\{f_{1}-\mu_{2}, \mu_{4}\right\}, \text { where } \\
f_{1} & =I\left(X_{1} ; Y_{3} \mid U_{2}, X_{3}\right)+I\left(X_{1}, U_{2}, X_{3}, Y_{3} ; X_{4}, \hat{Y}_{4}\right),
\end{aligned}
$$

Comparing with $A_{2}$ in (11), we need to show that $f_{1}=\mu_{12}$. Lets start with $f_{1}$

$$
\begin{aligned}
f_{1} & =I\left(X_{1} ; Y_{3} \mid U_{2}, X_{3}\right)+I\left(X_{1}, U_{2}, X_{3}, Y_{3} ; X_{4}, \hat{Y}_{4}\right) \\
& =I\left(X_{1} ; Y_{3} \mid U_{2}, X_{3}\right)+I\left(X_{1}, U_{2}, X_{3}, Y_{3} ; X_{4}\right) \\
& +I\left(X_{1}, U_{2}, X_{3}, Y_{3} ; \hat{Y}_{4} \mid X_{4}\right) \\
& \stackrel{a}{=}
\end{aligned}
$$

where $(a)$ follows since $X_{4}$ is independent from $\left(X_{1}, U_{3}, X_{3}\right)$ as in the input distribution in (3). The equivalency of the other constraints can be proved in a similar way.

\section{REFERENCES}

[1] I.-H. Wang and D. Tse, "Interference mitigation through limited receiver/ transmitter cooperation," IEEE Trans. Info. Theory, vol. 57, no. 5, pp. 2913 -2965, may 2011.

[2] S. Yang and D. Tuninetti, "Interference channel with generalized feedback (a.k.a. with source cooperation): Part I: Achievable region," IEEE Trans. on Info. Theory, vol. 57, no. 5, pp. 2686-2710, May 2011.

[3] V. Prabhakaran and P. Viswanath, "Interference channels with source/ destination cooperation," IEEE Trans. Info. Theory, vol. 57, no. 1, pp. $156-209$, jan. 2011.

[4] Y. Cao and B. Chen, "An achievable rate region for interference channels with conferencing," in Proc. IEEE Int'l Symp. on Info. Theory (ISIT), June 2007, pp. 1251-1255.

[5] A. Host-Madsen, "Capacity bounds for cooperative diversity," IEEE Trans. on Info. Theory, vol. 52, no. 4, pp. 1522-1544, Apr. 2006.

[6] H. Do, T. Oechtering, and M. Skoglund, "Noisy network coding approach to the interference channel with receiver cooperation," in Comm., Control, and Computing (Allerton), 2011 49th Annual Allerton Conf. on, sept. 2011, pp. $839-846$.

[7] A. E. Gamal and Y.-H. Kim, Network Information Theory, 1st ed. Cambridge University Press, 2011.

[8] S. H. Lim, Y.-H. Kim, A. El Gamal, and S.-Y. Chung, "Noisy network coding," IEEE Trans. on Information Theory, vol. 57, no. 5, pp. 31323152, May 2011.

[9] P. Zhong, A. Abu Al Haija, and M. Vu, "On compress-forward without wyner-ziv binning for relay networks," submitted to IEEE Trans. on Info. Theory, July 2011, available at http://arxiv.org/abs/1111.2837v1.

[10] T. Cover and A. Gamal, "Capacity theorems for the relay channel," IEEE Trans. on Info. Theory, vol. 25, no. 5, pp. 572-584, Sep. 1979.

[11] A. El Gamal, M. Mohseni, and S. Zahedi, "Bounds on capacity and minimum energy-per-bit for awgn relay channels," Information Theory, IEEE Transactions on, vol. 52, no. 4, pp. 1545 -1561, April 2006. 